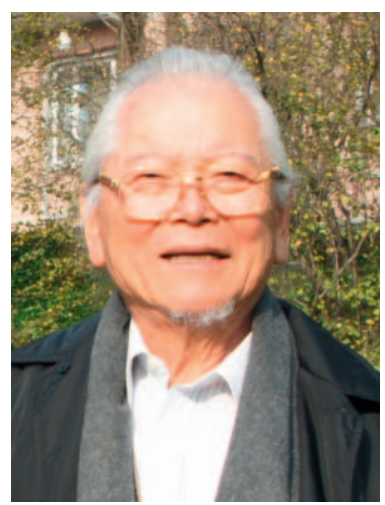

\title{
Interview mit Dr. med. Toshikatsu Yamamoto, dem Grand Seigneur der Akupunktur
}

Dr. med. Toshikatsu Yamamoto ist eine lebende Legende in der Akupunkturszene. Weltweite Bedeutung erlangte der 82-Jährige durch die Entwicklung der «Neuen Schädelakupunktur nach Yamamoto» (YNSA). 15000 Ärzte hat er bisher ausgebildet - jährlich wird seine Methode weltweit 6 Millionen Mal angewendet. Noch heute behandelt der Grand Seigneur täglich 60 Patienten. Anlässlich seines Besuchs in der Aeskulap-Klinik in Brunnen (Abb. 1 und 2), die auch als Zentrum für diese Akupunkturmethode gilt, sprach Dr. Josef Roos mit dem Arzt.

Sie haben die Yamamoto-Schädelakupunktur entwickelt und sind eine lebende Legende. Wie haben Sie es zu diesem Ruhm gebracht?

Meine Arbeit mache ich einfach mit Begeisterung. Was ich betreibe, lebe ich. Ich suchte ganz einfach Wege, den Patienten zu helfen.

Sie wurden dieses Jahr bereits 82 Jahre alt und sind immer noch so aktiv wie ein 40-Jähriger. Wie schaffen Sie das?

Ich arbeite mit Begeisterung und geniesse das Leben. Arbeit ist für mich Hobby und Lebenserfüllung zugleich. Tatkräftige Unterstützung bekomme ich jedoch von meiner Frau. Denn alleine geht es nicht.

Wie viele Patienten behandeln Sie immer noch pro Tag?

60 Patienten. Fünf Tage in der Woche - eigentlich 52 Wochen pro Jahr. Auch in meinen Seminaren behandele ich Patienten.
Sie sind ein begeisterter Arzt. Was macht die Faszination Ihrer Tätigkeit für Sie aus?

Es gibt nichts Schöneres, als Menschen zu helfen und bessere Behandlungsmöglichkeiten $\mathrm{zu}$ entwickeln. Mein Forschergeist ist ungebrochen. Das, was andere machen und gefunden haben, interessiert mich weniger. Ich möchte neue Sachen kreieren.

Wie schaffen Sie es, die Menschen weltweit mit Ihrer Art in den Bann zu ziehen?

Ich lebe vor, was ich mache. Die Menschen bemerken, dass ich Arzt geworden bin, weil ich ihnen helfen möchte. Ich freue mich bei jedem Patienten, wenn es ihm besser geht.

Welche Ziele und Perspektiven haben Sie noch? Was wollen Sie noch verwirklichen?

Noch lange zu leben und Menschen zu helfen.
Weshalb entschieden Sie sich, Arzt zu werden?

Eigentlich sollte ich von meinem Vater aus Landwirt oder Förster werden. Da damals Landwirte und Förster erst um $21 \mathrm{Uhr}$ assen, hätte ich immer Hunger gehabt. Lehrer und Ärzte bekamen schon um 18 Uhr etwas zu essen. Heute ist es aber so, dass Landwirte schon um $18 \mathrm{Uhr}$ essen, während ich noch lange Zeit am Patienten arbeite. Normale Essenszeit heute: $21 \mathrm{Uhr}$.

Und weshalb entschieden Sie sich, sich auf Akupunktur zu spezialisieren? Aus Versehen - oder durch Zufall?

Als westlich ausgebildeter Arzt habe ich in meiner Ausbildung und auch vorher keine Akupunkturkenntnisse gehabt. Ich habe als Anästhesist und Schmerztherapeut bei einer Patientin eine Nervenblockade nur mit destilliertem Wasser behandelt, da ich vergessen hatte, das Lokalanästhetikum mit aufzuziehen. Die Injektion war sehr schmerzhaft. Aber am nächsten Tag berichtete die Patientin über einen

\section{KARGER}

Fax +49761 4520714 Information@Karger.d Information@Kar
(๑) 2012 S. Karger GmbH, Freiburg www.karger.com/szg
Dr. Josef Roos

Medienverantwortlicher Aeskulap-Klinik Gersauerstrasse 8, 6440 Brunnen, Schweiz

Tel. +41 41 825-4747, Fax -4965 josef.roos@aeskulap.com 


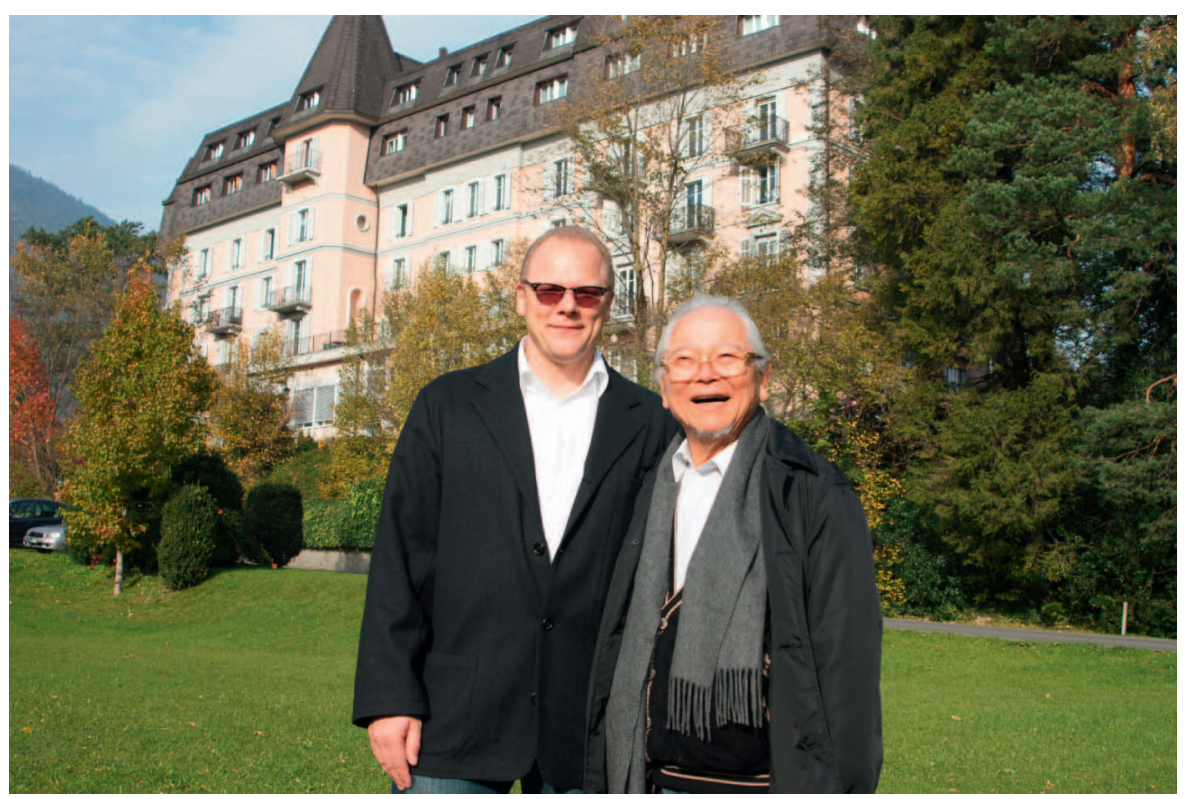

Abb. 1. Dr. Hans Peter Ogal und Dr. Toshikatsu Yamamoto in Brunnen.

Rückgang ihrer Schmerzen und «komische» Ausstrahlungen über den Körper. Da ich mir dies anatomisch nicht erklären konnte, habe ich mir ein Buch über die Meridiane ausgeliehen und erstmals Kontakt zur Akupunktur und zur Lehre der Traditionellen Chinesischen Medizin (TCM) bekommen.

Nachdem ich viele Schlaganfallpatienten mit Lähmungen behandelt habe, interessierte ich mich für die chinesische Schädelakupunktur; jedoch waren hier meine Erfolge nicht so gut. Durch Zufall kam ich auf effektivere Punkte am Kopf und experimentierte mit diesen. Hier begann die Geschichte der YNSA.

Sie sind Jahrgang 1929. Welche Erinnerungen haben Sie an die Zeit des Zweiten Weltkriegs und Hiroshima?

Ich war noch so klein. In dieser Zeit gab es wenige Informationen - kein Radio, keine Zeitung. Eigentlich sollte ich freiwillig Kamikaze-Pilot werden, wollte aber nicht, da ich und meine Mutter Angst hatten. Für meinen Vater wäre dies jedoch eine Ehre gewesen. Zum Glück bin ich aber nicht gegangen.
Eigentlich viel schwerer; die Ausbildung war viel begrenzter, und dann folgte eine deutlich höhere Arbeitsbelastung als heutzutage.

Weshalb entschieden Sie sich anfänglich, sich auf Anästhesie, Chirurgie und Geburtshilfe zu spezialisieren?

Erst wollte ich Anästhesieprofessor werden, da es so wenige Anästhesisten gab. Ich habe meine Anästhesieausbildung in den USA gemacht und wollte dann in Köln Gynäkologie lernen. Da mich der gynäkologische Chef auch als Anästhesist brauchte, musste ich nicht erst einmal ein halbes Jahr ohne Bezahlung arbeiten, sondern konnte direkt auch als Japaner eine gynäkologische Ausbildung machen.

Eigentlich hatte ich gar nicht vor, eine Praxis aufzumachen. Ich habe auch das Kassensystem in Japan nicht kennen gelernt. Trotzdem bin ich mit meiner neuen Familie in meine Heimat und habe eine Praxis eröffnet, da es im südjapanischen Nichinan kaum Ärzte gab. Aus der Praxis wurde schnell eine Klinik, und später kamen noch ein grosses Rehabilitationscenter in Miyazaki und zwei Altersheime dazu.

Wie erlebten Sie die Nachkriegszeit von 1945 bis 1955 in Japan als junger Student. Welche Möglichkeiten bot man Ihnen damals?

Nach dem Studium bin ich 1956 nach New Jersey gegangen, um meine medizinische Ausbildung zu ergänzen und die Welt kennen zu lernen. Ich lernte die ganze Welt kennen - und meine Frau, die mich mein ganzes Leben unterstützt hat. Sie ist in Deutschland geboren, lebte nach dem Krieg in England und hat dann die englische Nationalität erworben. Somit haben wir viel von der Welt kennen gelernt.

War es damals einfacher als heute, Arzt zu werden und den Beruf auszuüben?

\section{Wieso Altersheime?}

Ich kann so auch meine «älteren» Patienten direkt weiterbetreuen.

Was bewog Sie, Ihre Facharztausbildung in den USA zu machen und danach in Deutschland zu arbeiten?

Während meiner Studienzeit lernte ich einen Professor kennen, der aus den USA kam. Dieser hatte neue medizinische Zusammenhänge gelernt, die mich sehr interessierten. So beschloss ich, unbedingt meine Ausbildung in den USA zu erweitern. 
Wie erlebten Sie damals Deutschland? Welche Möglichkeiten bot man Ihnen damals?

In den USA gab es in der Medizin keine hierarchischen Unterschiede. Alle waren gleich. In Deutschland gab es diese noch sehr ausgeprägt.

Für welche Patienten ist Ihre Methode besonders sinnvoll?

Für Schmerzpatienten mit den unterschiedlichsten Ursachen und bei Lähmungen. Prinzipiell gilt: Erst einmal versuchen und dem eigenen Können keine Grenzen setzen.

Weshalb ist Ihre Methode besonders für Schmerzpatienten sinnvoll?

Weil sie schnell (meist sofort) wirkt und auch dann häufig noch wirkt, wenn Patienten schon viele Behandlungsmethoden vergeblich versucht haben.

Wie werden die einzelnen Punkte am Schädel bei Ihrer Methode ermittelt?

Über die diagnostischen Areale am Bauch und am Hals. Diese weisen auf die zu behandelnden Punkte am Kopf hin. Am Kopf können die Punkte auch palpiert werden, da sie bei länger bestehenden Störungen trophische Veränderungen aufweisen.

Wie haben Sie dies eigentlich herausgefunden?

Ganz zufällig. Wie vieles im Leben ...

Wie lange braucht man, damit man Ihre Methode beherrscht?

In den Grundzügen kann man die YNSA sehr schnell erlernen; bei schwierigen Krankheitsbildern

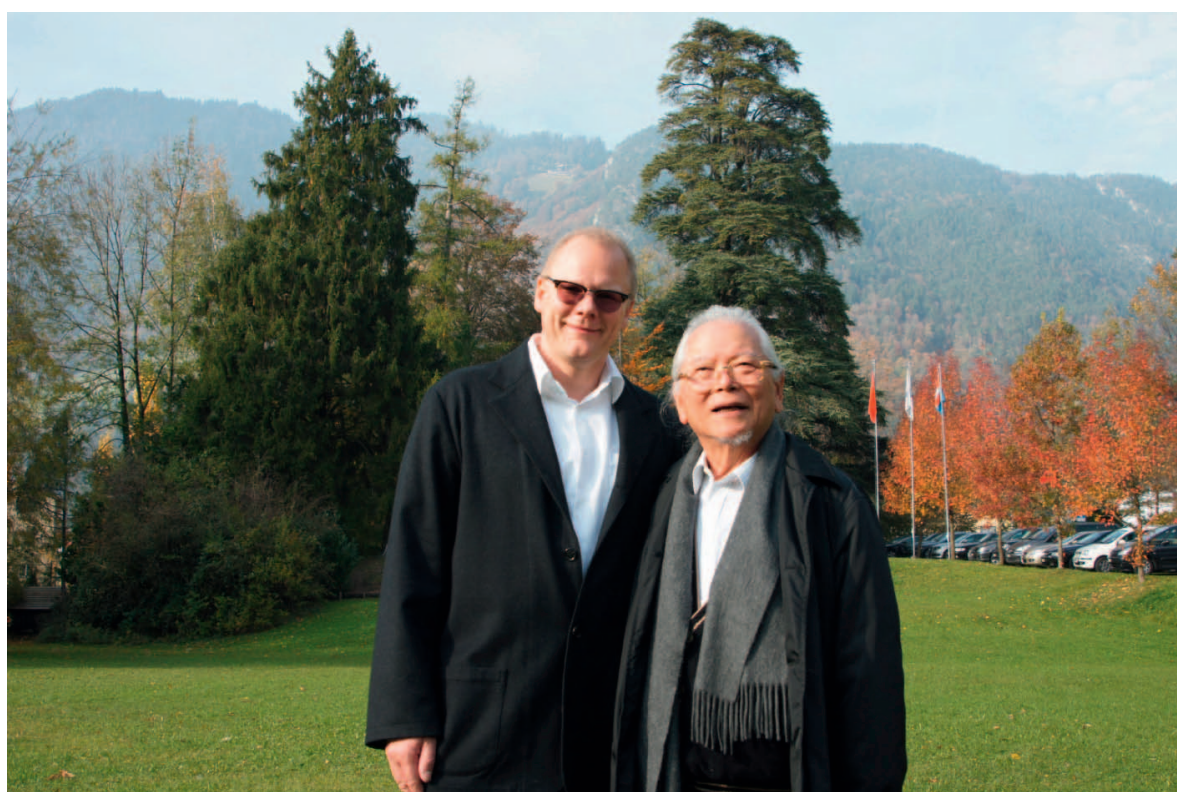

Abb. 2. Dr. Hans Peter Ogal und Dr. Toshikatsu Yamamoto in Brunnen.

braucht man wie bei jeder Methode Übung, Routine und Forschergeist.

Weshalb muss eine Zusatzausbildung gemacht werden, um Ihre Methode auszü̈ben?

Meine Methode unterscheidet sich voll und ganz von der TCM.

Sie unterrichten noch heute. Was macht die Faszination dieser Tätigkeit aus und wie lange möchten Sie dies noch machen?

Meine Zufriedenheit ist es, Menschen $\mathrm{zu}$ helfen. Wenn ich Kollegen meine Methode beibringe, erweitert sich die Anzahl der Patienten, denen wir damit helfen können. Ich werde so lange weitermachen, bis ich nicht mehr kann.

Inwiefern hatten Sie nebst Ihrem riesigen beruflichen Engagement noch ein Privatleben?

Ich lebe für meinen Beruf und hatte grosses Glück, dass meine Frau Krankenschwester ist und somit Verständ- nis für mein Engagement für die Patienten hat und dies unterstützt.

Welche Beziehung haben Sie zur Schweiz?

Ich fühle mich in der Schweiz sehr wohl und schätze die persönlichen Beziehungen zu Hans Ogal, dem Leiter der Schmerzabteilung in der Aeskulap-Klinik, und seiner Frau Mercedes. Er war vor genau 20 Jahren bei uns zu Hause und in der Klinik. Er konnte die YNSA sehr gut lernen, hat auch ein Buch darüber geschrieben, und wir halten heute zusammen Kurse und Weiterbildungen.

In welchen Ländern halten Sie Weiterbildungen über die YNSA?

USA, Brasilien, Deutschland, Italien, Spanien, Ungarn, Rumänien, Bulgarien, England, Russland, Schweden, Dänemark, Ägypten, Australien, Holland, Österreich, Polen, Tschechien, Slowakei, Türkei, Sri Lanka, Taiwan, Korea, China, Griechenland, Japan und andere. 
Sie hielten mit Dr. Ogal aktuell Spezialistenkurse in der Hochschule Hannover und bei der Medizinischen Woche in Baden-Baden. Dort werden auch Patienten mit sonst nicht mehr behandelbaren Erkrankungen behandelt (z.B. Lähmungen nach Apoplex und Traumata, Lähmungen bei Kindern nach Geburtstrauma, Schwindel seit Jahren, schwerste chronifizierte Schmerzsymptomatiken, neurologische Störungen). Die Ergebnisse sind so überraschend gut, dass viele Patienten Tränen in den Augen haben. Wieso hilft hier die YNSA?

Sicher über die Behandlung des zentralen Nervensystems und den direkten Zugang zu diesem über die YNSA-Punkte. Auch bei langjährigen Beschwerden können häufig noch gute Ergebnisse erzielt werden. Gerne würde ich empfehlen: «Erst mal ver- suchen, bevor man sagt, «man könne nichts mehr machen.$»$

Wie lange sollte man behandeln?

Das ist ganz individuell.

Sollten die Patienten zuerst zur Behandlung zu Ihnen nach Japan kommen?

Das ist $\mathrm{zu}$ weit weg. Sie sollten sich einen guten Arzt, der die YNSA beherrscht, in ihrer Nähe suchen.

\section{Ist die YNSA teuer?}

Nein, keineswegs. Der Arzt braucht hierfür eine gute Ausbildung, aber die Anwendung am Patienten muss nicht teuer sein. Ganz im Gegenteil. Da z.B. einige Ärzte in der Provinz São Paulo in Brasilien YNSA anwenden, konnten die Gesundheitskosten in der ganzen Provinz um 12\% reduziert werden.

Machen Sie mit Ihrer Frau auch mal Ferien?

Eigentlich viel zu selten und zu kurz. Unsere längsten echten Ferien waren nicht länger als 3-4 Tage. Letzte Woche durften wir uns mal 4 Tage in der Schweiz erholen und die Gastfreundschaft in Brunnen unter anderem mit einem Besuch des Männerchors geniessen. Dies haben wir unseren Töchtern $\mathrm{zu}$ verdanken, die in der Zeit unserer Abwesenheit die Patienten in der Klink weiterbehandeln und unsere Unternehmungen weiterführen.

Interview: Josef Roos 